\title{
Evaluation of Tension, Bending and Twisting Rigidities of Single-Layer Graphene Sheets by an Analytical Asymptotic Homogenization Model
}

\author{
Sobhan SAFARIAN*, Masoud TAHANI** \\ *Department of Mechanical Engineering, Faculty of Engineering, Ferdowsi University of Mashhad, \\ P.O. Box 91775-1111, Mashhad, Iran, E-mail: safarian.sobhan@gmail.com \\ **Department of Mechanical Engineering, Faculty of Engineering, Ferdowsi University of Mashhad, \\ P.O.Box91775-1111, Mashhad, Iran, E-mail: mtahani@um.ac.ir

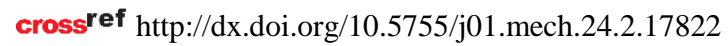

\section{Introduction}

To develop novel materials such as graphene sheets and carbon nanotubes (CNTs) too many studies are in progress in this modern field of research [1]. The singlelayer graphene sheet (SLGS) is comprised of a monolayer of carbon atoms arranged periodically and uniformly in a hexagonal honeycomb mode. The hexagonal nanostructure of SLGS with the high strength of the carbon-carbon covalent bond creates a strong material, two hundred times harder than steel [2].

SLGS shows several desirable physical properties, such as small size, low density, high strength and excellent electrical and thermal properties. These exceptional mechanical and physical properties along with recent advances in synthesis methods and their purification make graphene sheet becomes an excellent candidate for use in composite reinforcement. To find out the potential of SLGS as reinforcement in composite materials, a proper understanding of their mechanical properties is essential. Since the experimental measurement of mechanical properties of graphene-based materials is a troublesome task, there are a few distinctive reported works in the literature. For example, Lee et al. [2] obtained Young's modulus of $1 \mathrm{TPa}$ for monolayer defect-free graphene, by nanoindentation using an atomic force microscope. Frank et al. [3] measured Young's modulus of suspended graphene sheets equal to $0.5 \mathrm{TPa}$.

Theoretical studies on the effective properties of SLGSs are essentially based on two different approaches: the continuum mechanics approach and atomistic modeling methods [4-9]. Atomistic modeling is computationally intensive, hence the application is limited to small numbers of atoms. On the other hand, substitution of a network molecular structure with a continuum media was a challenging task.

Lu et al. [4] proposed a new formula for elastic flexural modulus of single-layer graphene sheet based on an empirical potential for carbon atoms in the solid state. Tsai and $\mathrm{Tu}$ [5] estimated the mechanical properties of graphite in the forms of single graphene layer and graphite flakes using molecular dynamics (MD) simulation. Sakhaee-Pour [6] proposed a beam based atomistic simulation method to compute the elastic characteristics of defect-free SLGS. Scarpa et al. [7] used beam type models to describe the in-plane linear elastic properties of SLGSs. Georgantzinos et al. [8] studied single- and multi-layer graphene structures using structural mechanics atomistic modeling with spring-like finite elements. Mir at al. [9] established, a nanoscale analytical continuum theory for determination of stiffness and
Young's modulus of carbon nanotubes. They observed no diameter dependence for Young's modulus of CNTs. They obtained the value of Young's modulus of CNT as $1.42 \mathrm{TPa}$ and $1.30 \mathrm{TPa}$ for zigzag and armchair CNTs, respectively.

This paper studies the effective mechanical properties of single-layered graphene sheets by analytical asymptotic homogenization method which is focused on the linear elasticity. Because of heavy atomistic simulation on the nanostructures, many researchers have been interested in the connection between nano/microstructures and the classical continuum mechanics. This paper is also in this category. Grafting the asymptotic homogenization approach onto the single-layered graphene sheets problem is a new try. The merit of using asymptotic homogenization model is that single-layered graphene sheet as a periodic nanostructure could be modeled by asymptotic homogenization method with low computational cost regardless of dimensions of the model. The approach is an analytical one and makes use of asymptotic homogenization techniques and goes around atomistic simulations to develop constitutive relations for predicting the effective elastic properties of the single-layer graphene sheet. These effective properties are estimated from the solution of the proper local problem on the unit cell.

Here we employ both interatomic interactions based on bond stretching and bond bending forces in the asymptotic homogenization model. The influence of force field constants on the elastic properties of SLGSs and the importance of choosing them for $\mathrm{C}-\mathrm{C}$ bonds are examined in three different types of models which are purposed for atomic potentials. It is found that the force field constants significantly affect the effective elastic properties and rigidities of the graphene sheet.

\section{Asymptotic homogenization method (AHM)}

\subsection{A brief review of asymptotic homogenization}

The most capable, viable and graceful technique for linking the macroscopic fields in periodic nanofield models is based on a mathematical theoretical account referred to as asymptotic homogenization, asymptotic expansion homogenization, or homogenization theory. In mathematics, we have a limitation in applying the theory of homogenization which uses the asymptotic expansion and assumes periodicity. Up to now, Kalamkarov et al. [10,11] and some other researchers $[12,13]$ have used homogenization techniques to derive effective elastic, piezoelectric and thermal expansion coefficients in materials. 
Here, from a nano-mechanical point of view, we assume that the covalent bond between carbon atoms can be represented by reinforcements. The discrete periodic arrangements of the SLGS which is modeled by a honeycomb network sheet with a hexagonal unit cell structure convinces us to use asymptotic homogenization method. The sheet, in turn, can be used as a thin homogeneous layer of reinforced composite with matrix around the circumference of the reinforcements (Fig. 1). The reinforcements (covalent bonds) are much stronger than their matrix, so the circumference of the reinforcements is discounted in the analysis. It means that the surrounding matrix is modeled by assuming zero stiffness.

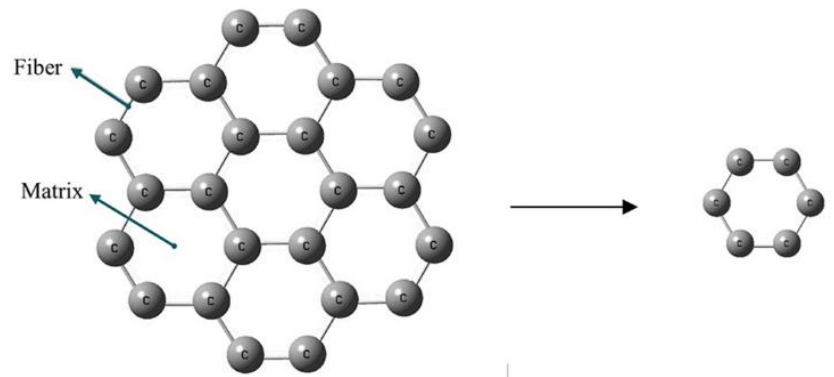

Fig. 1 A model of a discrete periodic lattice structure of SLGS with the equilateral hexagonal unit cell.

2.2. Governing equations of the asymptotic homogenization for composite structure

A heterogeneous composite structure is said to have a regular periodicity while a composite tissued by reinforcements that arranged in a particular kind with a periodically repeating cell Y (Fig. 2). In the theory of homogenization, in comparison to the dimensions of the overall domain, $\mathrm{Y}$ is assumed to be quite little. In the asymptotic homogenization method double scales for studying of all quantities are considered: one on the global level $x$ and the other on the local level $y$ which are so-called slow and fast variables, respectively. A small parameter $\delta$ defines the ratio of the real length of a unit vector $x=\left(x_{1}, x_{2}, x_{3}\right)$ in the global coordinates to the real length of a unit vector $y=\left(y_{1}, y_{2}, y_{3}\right)$ in the local coordinates, so $\delta y=x$ or $y=x / \delta$ [10-12].

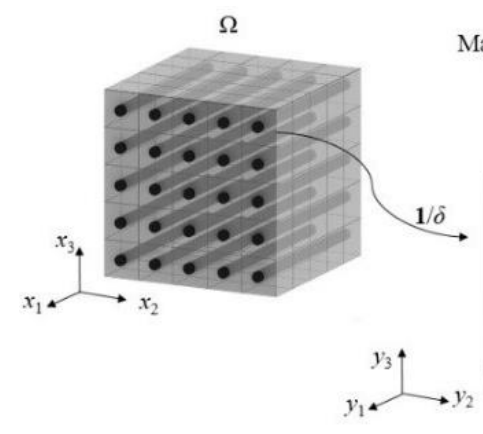

a

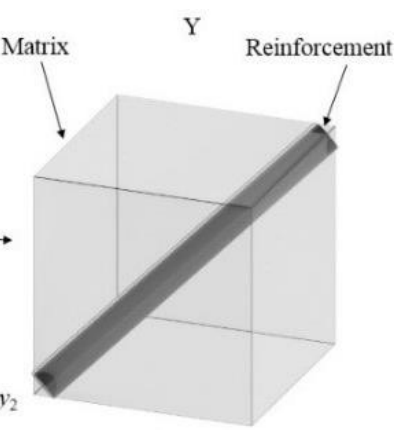

b
Fig. 2 (a) A heterogeneous composite structure and (b) periodicity (unit) cell

The governing equation, in the form of equilibrium equation in the absence of body forces is [14]:

$$
\frac{\partial \sigma_{i j}^{\delta}}{\partial x_{i}}=0 \text { in } \Omega, u^{\delta}(x)=0 \text { on } \partial \Omega,
$$

where:

$$
\sigma_{i j}^{\delta}(x, y)=C_{i j k l}\left[\frac{1}{2}\left(\frac{\partial u_{k}^{\delta}(x, y)}{\partial x_{l}}+\frac{\partial u_{l}^{\delta}(x, y)}{\partial x_{k}}\right)\right],
$$

with:

$$
C_{i j k l}\left(x, \frac{x}{\delta}\right)=C_{i j k l}\left(\frac{x}{\delta}\right)=C_{i j k l}(y),
$$

where in the above equations $C_{i j k l}$ is the tensor of elastic coefficients and $u_{i}$ is the component of displacement field. As a result, if $\varphi(x, y)=\varphi(x, x / \delta)$ is a general function then according to rules of differentiation we have:

$$
\frac{d \varphi}{d x}=\frac{\partial \varphi}{\partial x_{i}}+\frac{\partial y_{i}}{\partial x_{i}} \frac{\partial \varphi}{\partial y_{i}}=\frac{\partial \varphi}{\partial x_{i}}+\frac{1}{\delta} \frac{\partial \varphi}{\partial y_{i}} .
$$

The displacement and stress fields are expanded using the scale asymptotic expansion in terms of the small parameter $\delta$ as:

$$
\left\{\begin{array}{c}
u^{\delta}=u^{(0)}+\delta u^{(1)}+\delta^{2} u^{(2)}+\ldots . \\
\sigma_{i j}^{\delta}=\sigma_{i j}^{(0)}+\delta \sigma_{i j}^{(1)}+\delta^{2} \sigma_{i j}^{(2)}+\ldots .
\end{array}\right.
$$

where $u^{(i)}(x, y)$ and $\sigma^{(i)}(x, y)$ are periodic on $y$. Using the latter fact in Eq. (4), by separating $x_{i}$ and $y_{i}$ and afterward, by substituting Eqs. (5) into Eqs. (1) and (2), with equating the terms with the zero power of $\delta$ we get:

$$
\frac{\partial}{\partial y_{j}}\left[C_{i j k l}(y)\left(\frac{\partial u_{k}^{(1)}(x, y)}{\partial y_{l}}+\frac{\partial u_{k}^{(0)}(x)}{\partial x_{l}}\right)\right]=0,
$$

and by comparing other terms with the same power of $\delta$ it can easily be shown that $u^{(0)}$ and $\sigma^{(0)}$ are independent of the local variable $y$ and only depend on $x$. By separating $x_{i}$ and $y_{i}$ in Eq. (6) we decide that $u^{(1)}$ has the following form:

$$
u_{n}^{(1)}(x, y)=\frac{\partial u_{k}^{(0)}(x)}{\partial x_{l}} \chi_{n}^{k l}(y)+\xi_{n}(x)
$$

where: $\chi(y)$ is a function that is periodic in $y$ and satisfies:

$$
\frac{\partial}{\partial y_{j}}\left[C_{i j k l}(y)\left(\frac{\partial \chi_{m}^{k l}(y)}{\partial y_{n}}+1\right)\right]=0
$$

and $\xi(x)$ is a function of $x$ which is constant of integration from $y$. Eq. (8) depends only on the rapid variable $y$ and therefore this equation is called the unit cell of the problem. By integrating Eq. (8) over the range of the unit cell Y (by volume $|\mathrm{Y}|$ ) which defines according to: 


$$
\langle f\rangle=\frac{1}{|\mathrm{Y}|} \int f\left(y_{1}, y_{2}, y_{3}\right) d y_{1} d y_{2} d y_{3}
$$

and the following result is achieved:

$$
\tilde{C}_{i j k l}=\left\langle C_{i j k l}(y)+C_{i j m n}(y) \frac{\partial \chi_{m}^{k l}}{\partial y_{n}}\right\rangle,
$$

where: $\tilde{C}_{i j k l}$ 's are the effective elastic coefficients. The effective elastic coefficients are found when the unit cell problem in (8) is solved and the functions $\chi_{m}^{k l}$ are determined $[10,11]$.

Now, a general macroscopically composite structure with a number of components as reinforcements is assumed. The effective elastic coefficients for composite structures with several groups of reinforcements are calculated by using the principle of superposition [10,11]. Each reinforcement or bar is oriented with its local orthogonal coordinates $\eta_{1}, \eta_{2}$ and $\eta_{3}$. Before doing anything with the data, the original coordinates $y_{1}, y_{2}$ and $y_{3}$ should be transformed to local orthogonal coordinates $\eta_{1}, \eta_{2}$ and $\eta_{3}$ (Fig. 3). The new coordinates are recounted as the $\eta_{1}$ coordinate axis coincides with the direction of the reinforcement axis and $\eta_{2}$ and $\eta_{3}$ are perpendicular to it and perpendicular to each other. Thus, the derivatives transform according to $\partial / \partial y_{i}=Q_{j i} \partial / \partial \eta_{j}$ where the components of the matrix $Q_{j i}$ 's are direction cosines characterizing the rotation axis.

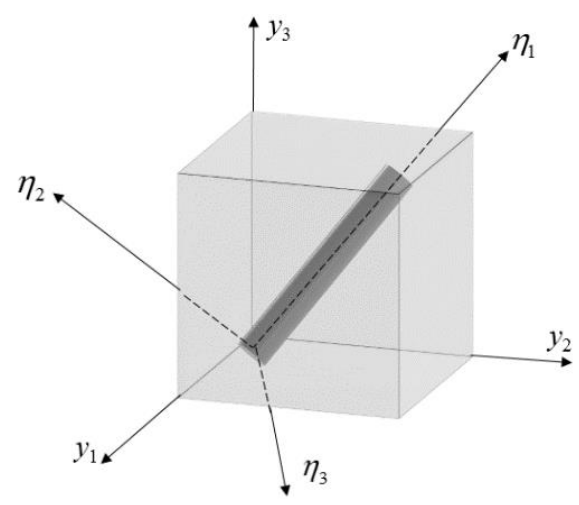

Fig. 3 The unit cell of composite reinforced with single reinforcement in original and rotated coordinates

From Eq. (9), the function $B_{i j}^{k l}$ for the unit cell problem is introduced by the following equation:

$$
B_{i j}^{k l}=C_{i j m n} \frac{\partial \chi_{m}^{k l}(y)}{\partial y_{n}}+C_{i j k l} .
$$

From interfacial continuity conditions that exist between the matrix and the reinforcements, both conditions for the function $\chi_{m}^{k l}(y)$ are $\left.\chi_{n}^{k l}(r)\right|_{s}=\left.\chi_{n}^{k l}(m)\right|_{s}$ and $\left.B_{i j}^{k l} n_{j}(r)\right|_{s}=\left.B_{i j}^{k l} n_{j}(m)\right|_{s}$ where the indices $s, r$ and $m$ show the fiber matrix interface, reinforcement and matrix, respectively and $n_{j}$ are the components of the unit normal vector at the interface $[10,11]$.

\subsection{Asymptotic homogenization for a SLGS}

As mentioned earlier since SLGS can be considered as a thin homogeneous layer of the matrix in the hole, here we assume the matrix is "soft" and take $B_{i j}^{k l}(m) \approx 0$. Covalent bonds between carbon atoms can be represented by reinforcements, where the fibers are much stiffer than matrix in the graphene sheet, so in the simplest model for SLGS, the condition becomes $\left.B_{i j}^{k l} n_{j}(r)\right|_{s}=0$. Finally, from Eq. (8) with continuity conditions, the problem that must be solved for a single isotropic bar becomes:

$$
\frac{\partial B_{i j}^{k l}}{\partial y_{j}}=0,\left.\quad B_{i j}^{k l} n_{j}(r)\right|_{s}=0 .
$$

Supposing the length, the cross-sectional area of the reinforcement and volume of the unit cell are $L, A$ and $V$, respectively, then the effective elastic homogenized coefficients which are obtained through integration of $B_{i j}^{k l}$ over the volume of the entire unit cell are:

$$
\left\langle B_{i j}^{k l}\right\rangle=\frac{1}{|\mathrm{Y}|} \int_{Y} B_{i j}^{k l} d v=\frac{A L}{V} B_{i j}^{k l}=V_{f} B_{i j}^{k l},
$$

where $V_{f}$ is the volume fraction of the reinforcement in the unit cell. The effective coefficients of SLGS with several groups of bars can be represented by:

$$
\left\langle B_{i j}^{k l}\right\rangle=\sum_{n=1}^{N} V_{f}^{(n)} B_{i j}^{(n) k l} .
$$

Therefore, in general homogenization of SLGS model the constitutive relations of the equivalent anisotropic homogeneous shell are given as:

$$
\begin{aligned}
& N_{i j}=\delta\left\langle B_{i j}^{k l}\right\rangle \varepsilon_{k l}+\delta^{2}\left\langle B_{i j}^{* k l}\right\rangle \kappa_{k l}, \\
& M_{i j}=\delta^{2}\left\langle z B_{i j}^{k l}\right\rangle \varepsilon_{k l}+\delta^{3}\left\langle z B_{i j}^{* k l}\right\rangle \kappa_{k l},
\end{aligned}
$$

where $N_{i j}, M_{i j}, \varepsilon_{k l}$ and $\kappa_{k l}$ are the stress resultants, moment resultants, mid-surface strains and curvatures, respectively. A plane stress problem is assumed for the homogeneous shell which is a thin planar structure with constant thickness and loading within the plane of the structure. Thus the indices such as $i, j, k$ and $l$ could take values 1 and 2, whereas $\delta$ is defined as the thickness of shell which is quite thin in comparing with the two other dimensions of the sheet. For the purposes of SLGS, we investigate our model for the case of 2D network honeycomb shell whereas the reinforcements lie in the $\alpha_{1}-\alpha_{2}$ plane. Fig. 4 shows the pertinent hexagonal unit cell for such a structure. Here for each bond as a reinforcement $z=\eta_{3}$ is perpendicular to the $\alpha_{1}-\alpha_{2}$ plane where $\eta_{1}$ and $\eta_{2}$ are in the $\alpha_{1}-\alpha_{2}$ plane, 
where $\alpha_{1}$ and $\alpha_{2}$ indicate zigzag and armchair directions, respectively. The quantities $\left\langle B_{i j}^{k l}\right\rangle,\left\langle B_{i j}^{* k l}\right\rangle,\left\langle z B_{i j}^{k l}\right\rangle$ and $\left\langle z B_{i j}^{* k l}\right\rangle$ are called the effective elastic coefficients and are integrated over the unit cell volume. Moreover, in Eqs. (15) and (16), $B_{i j}^{* k l}, z B_{i j}^{k l}$ and $z B_{i j}^{* k l}$ are defined as:

$$
\left\{\begin{array}{l}
B_{i j}^{* k l}=C_{i j m n}(\boldsymbol{y}) \frac{\partial \omega_{m}^{k l}(\boldsymbol{y})}{\partial y_{n}}+z C_{i j k l}, z B_{i j}^{k l}=z \cdot\left(B_{i j}^{k l}\right), \\
z B_{i j}^{* k l}=z C_{i j m n}(\boldsymbol{y}) \frac{\partial \omega_{m}^{k l}(\boldsymbol{y})}{\partial y_{n}}+z C_{i j k l}
\end{array}\right.
$$

where $\chi_{m}^{k l}(y)$ and $\omega_{m}^{k l}(y)$ are arbitrary linear functions of ( $\eta_{2}, \eta_{3}$ ) which should satisfy Eq. (12) for $B_{i j}^{k l}$ and $B_{i j}^{* k l}$, respectively.

Here we use both harmonic potential energy terms that are represented as $U_{r}=0.5 k_{r}(\Delta r)^{2}$ and $U_{\theta}=0.5 k_{\theta}(\Delta \theta)^{2}$ where $k_{r}$ and $k_{\theta}$ are the bond stretching and bond bending stiffness, respectively, while $\Delta r$ and $\Delta \theta$ represent bond stretching incrementation and bond angle variation, respectively [15]. The $a$ elements simulate the stretching with stiffness $k_{a}=k_{r}=E A / \ell$ for C-C bond interaction, while the $b$ elements represent the angle bending interaction with stiffness $k_{b}=E^{\prime} A^{\prime} / \ell^{\prime}$ (Fig. 5).

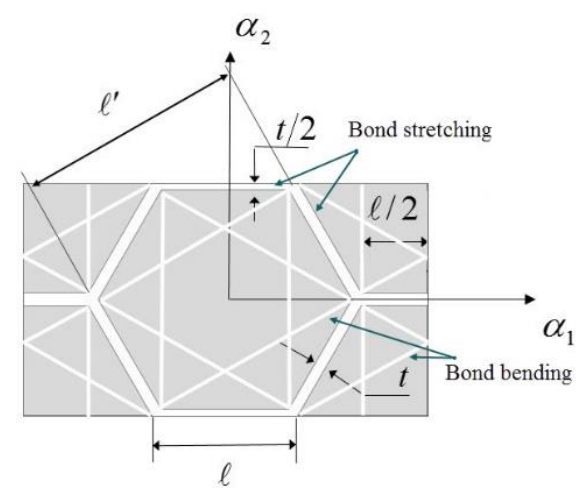

Fig. 4 Periodicity cell of SLGS considering both interatomic interactions due to bond stretching and bond bending

It should be noted that to simplify the model and make it more manageable, bending stiffness $k_{\theta}$ is replaced by a spring directly connected the opposite atoms with stiffness $k_{b}$ which is obtained by equalizing both energies of the straight and the torsion springs (Fig. 5). Giannopoulos et al. [15] stated that $k_{b}=(2 / \ell)^{2} k_{\theta}$. The stiffness values of $k_{a}$ and $k_{b}$ are used in the effective coefficients to the homogenization model of a hexagonal lattice structure. The length of bars or fibers which are simulated by elements $a$ and $b$ are represented by $\ell$ and $\ell^{\prime}$, respectively. It is assumed that the close carbon atoms are joined by a bar of the circular cross-section of diameter $\delta$ (Fig. 4). As shown in Fig. 4 this diameter $\delta$ could be assigned to the thickness of the hexagonal unit cell, it means that $\delta=t$ where $t$ is the thickness of the monolayer graphene sheet.

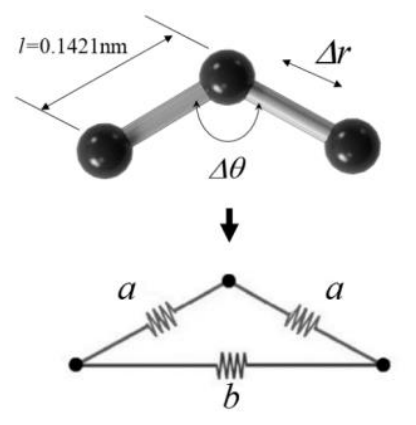

Fig. 5 Element $a$ is an extension spring and element $b$ is spring for bending resistance of a $\mathrm{C}-\mathrm{C}-\mathrm{C}$

Solving Eq. (12) for these elements by following the procedure from Eqs. (12)-(17) for the case of a SLGS gives the following expressions for the all nonzero effective elastic coefficients:

$$
\left\{\begin{array}{l}
\left\langle B_{\alpha \beta}^{\lambda \pi}\right\rangle=\sum_{j=1}^{N} k_{j} \Gamma_{j}^{\alpha \beta \lambda \pi} \gamma_{j},\left\langle z B_{\alpha \beta}^{\lambda \pi}\right\rangle=\left\langle B_{\alpha \beta}^{* \lambda \pi}\right\rangle=0, \\
\left\langle z B_{\alpha \beta}^{* \lambda \pi}\right\rangle=\sum_{j=1}^{N} k_{j}\left(\Gamma_{j}^{\alpha \beta \lambda \pi}+\frac{\Psi_{j}^{\alpha \beta \lambda \pi}}{1+v_{j}}\right) \frac{\gamma_{j}}{16},
\end{array}\right.
$$

where $v_{j}$ is Poisson's ratio of each bar and $\gamma_{j}$ is the volume fraction of reinforcement in the unit cell. The functions $\Gamma_{j}^{\alpha \beta \lambda \pi}$ and $\Psi_{j}^{\alpha \beta \lambda \pi}$ depend on the index combination $\alpha, \beta$, $\lambda$ and $\mu$ which are given as:

$$
\left\{\begin{array}{l}
\Gamma_{j}^{1111}=\frac{\ell}{A} \cos ^{4} \theta_{j}, \Gamma_{j}^{2222}=\frac{\ell}{A} \sin ^{4} \theta_{j}, \\
\Gamma_{j}^{1212}=\Gamma_{j}^{1122}=\Gamma_{j}^{2211}=\frac{\ell}{A} \cos ^{2} \theta_{j} \sin ^{2} \theta_{j} \\
\Psi_{j}^{1111}=\Psi_{j}^{2222}=-\Psi_{j}^{1122}=\frac{\ell}{A} \cos ^{2} \theta_{j} \sin ^{2} \theta_{j} \\
\Psi_{j}^{1212}=\frac{1}{4} \frac{\ell}{A}\left(\cos ^{2} \theta_{j}-\sin ^{2} \theta_{j}\right)^{2} \\
\Psi_{j}^{1112}=\frac{1}{2} \frac{\ell}{A}\left(\cos \theta_{j} \sin ^{3} \theta_{j}-\cos ^{3} \theta_{j} \sin \theta_{j}\right),
\end{array}\right.
$$

where $\Psi_{j}^{2211}=\Psi_{j}^{1122}, \quad \Psi_{j}^{2212}=\Psi_{j}^{1222}, \quad \Psi_{j}^{1211}=\Psi_{j}^{1112}$, $\Psi_{j}^{1222}=\Psi_{j}^{1112}$ and $\theta_{j}$ is the angle between the direction of $\eta_{1}$ for the $j$ th element of the unit cell and the $\alpha_{1}$ axis (see Figs. 3 and 4). Hence, by substituting $\left\langle B_{i j}^{k l}\right\rangle,\left\langle B_{i j}^{* k l}\right\rangle$, $\left\langle z B_{i j}^{k l}\right\rangle$ and $\left\langle z B_{i j}^{* k l}\right\rangle$ from Eqs. (18)-(19) into Eqs. (15)-(16), the stress resultants for SLGS are determined as:

$$
\left\{\begin{array}{l}
N_{11} \\
N_{22} \\
N_{12}
\end{array}\right\}=\left(\frac{1}{4 \sqrt{3}} k_{r}+2 \frac{k_{\theta}}{\sqrt{3} \ell^{2}}\right)\left[\begin{array}{lll}
3 & 1 & 0 \\
1 & 3 & 0 \\
0 & 0 & 1
\end{array}\right]\left\{\begin{array}{c}
\varepsilon_{11} \\
\varepsilon_{22} \\
2 \varepsilon_{12}
\end{array}\right\} .
$$

The results relevant to an armchair or zigzag direction in the unit cell can be obtained by interchanging indices 1 for 2 in Eq. (20) (Fig. 4). The elastic properties of homogenized SLGSs are gained from Eq. (20). It is noteworthy to 
highlight that in this work we employ both interatomic interactions due to bond stretching and bond bending forces and their stiffnesses $k_{r}$ and $k_{\theta}$ are applied in asymptotic homogenization model.

\section{Results and discussion}

To check the accuracy of the present asymptotic homogenization technique, some numerical results are presented for elastic properties of SLGSs. It should be pointed out that a carbon nanotube with a large diameter and high aspect ratio acts locally such as graphene. Therefore, if we ignore the effect of the curvature of CNTs, their unit cell problem will be comparable. Therefore, we could use similar models in which carbon nanotubes have been used in them. The plane stress constitutive relation from Eq. (15) for an orthotropic problem such as the graphene layer structures can be written as:

$$
\left\{\begin{array}{l}
\sigma_{11} \\
\sigma_{22} \\
\tau_{12}
\end{array}\right\}=\left[\begin{array}{ccc}
C_{11} & C_{12} & 0 \\
C_{21} & C_{22} & 0 \\
0 & 0 & C_{66}
\end{array}\right]\left\{\begin{array}{c}
\varepsilon_{11} \\
\varepsilon_{22} \\
\gamma_{12}
\end{array}\right\}
$$

where $C_{i j}$ 's are the elements of the stiffness matrix for plane stress problem. In Eq. (21) $\sigma_{i j}$ and $\varepsilon_{i j}$ are the stresses and strains, respectively, where stresses define as $\sigma_{11}=N_{11} / \delta, \quad \sigma_{22}=N_{22} / \delta$ and $\tau_{12}=N_{12} / \delta$. Inverting Eq. (21) gives the strain-stress relations as:

$$
\left\{\begin{array}{l}
\varepsilon_{11} \\
\varepsilon_{22} \\
\gamma_{12}
\end{array}\right\}=\left[\begin{array}{ccc}
S_{11} & S_{12} & 0 \\
S_{21} & S_{22} & 0 \\
0 & 0 & S_{66}
\end{array}\right]\left\{\begin{array}{l}
\sigma_{11} \\
\sigma_{22} \\
\tau_{12}
\end{array}\right\}
$$

where: $S_{i j}$ 's are the compliance coefficients, which are related to the engineering elastic constants. To obtain four independent compliance elements in the matrix, first, the values of $C_{i j}$ are determined by homogenization method. Then, the elastic modulus in the $\alpha_{1}$ direction can be calculated by applying a pure tensile load in direction $\alpha_{1}$. By definition, if the only nonzero stress is $\sigma_{11}$, then from Eq. (22), the effective modulus in $\alpha_{1}$ direction (zigzag direction, see Fig. 4) and Poisson's ratio $v_{12}$ are obtained as $E_{11}=\sigma_{11} / \varepsilon_{11}=1 / S_{11}$ and $v_{12}=-\varepsilon_{22} / \varepsilon_{11}=-S_{21} / S_{11}$ respectively. Similarly, the effective modulus towards $\alpha_{2}$ (armchair direction, see Fig. 4) and Poisson's ratio $v_{21}$ can be calculated by applying only nonzero stress $\sigma_{22}$ as $E_{22}=\sigma_{22} / \varepsilon_{22}=1 / S_{22}$ and $v_{21}=-\varepsilon_{11} / \varepsilon_{22}=-S_{12} / S_{11}$ respectively. Also, by applying a pure shear stress in the plane $1-2$, the effective shear modulus $G_{12}$ can be obtained as $G_{12}=\tau_{12} / \gamma_{12}=1 / S_{66}$. For SLGS, the strain-stress relation can be written as:

$$
\left\{\begin{array}{l}
\varepsilon_{11} \\
\varepsilon_{22} \\
\gamma_{12}
\end{array}\right\}=\frac{\delta}{\frac{1}{4 \sqrt{3}} k_{r}+2 \frac{k_{\theta}}{\sqrt{3} \ell^{2}}}\left[\begin{array}{lll}
3 & 1 & 0 \\
1 & 3 & 0 \\
0 & 0 & 1
\end{array}\right]^{-1}\left\{\begin{array}{c}
\sigma_{11} \\
\sigma_{22} \\
\tau_{12}
\end{array}\right\} .
$$

3.1. Effects of force field constants on elastic properties of SLGS

In this paper, a term $U_{\theta}$ is also added to consider the bond energy due to angle variation of bond bending $\Delta \theta$, which was neglected in earlier studies for homogenization model of CNT [14]. However, this term may play a more important role in mechanical properties of SLGS. Eqs. (20)(23) represent explicit relationships for the elastic properties of SLGS which depend only on force field constants $k_{r}$ and $k_{\theta}$ of C-C bonds from their atomic potentials and geometric parameters such as $\ell$, the length of C-C bonds and $\delta$, the diameter of C-C bonds or thickness of SLGS. Carbon-carbon bond length is considered to be $\ell=0.142 \mathrm{~nm}$ and we assume $t=\delta=0.34 \mathrm{~nm}$ in the calculations.

There are different functions available for describing the potential of $\mathrm{C}-\mathrm{C}$ bond interaction [16]. Force field constants $k_{r}$ and $k_{\theta}$ are found here by different atomic potentials in three types of models. By substituting $k_{r}$ and $k_{\theta}$ in Eq. (23) for three different types of force field constants from the different atomic potentials, the elastic properties of SLGS are obtained which are presented in Table 1 . In the model of type, I, we take the force field constants of Eqs. (23) equal to $k_{r}=6.52 \times 10^{-7} \mathrm{~N} / \mathrm{nm}$ and $k_{\theta}=8.67 \times 10^{-10} \mathrm{Nnm} / \mathrm{rad}^{2}$ from experimental data and empirical harmonic potential [15]. In type II, we use $k_{r}=7.42 \times 10^{-7} \mathrm{~N} / \mathrm{nm}$ and $k_{\theta}=14.2 \times 10^{-10} \mathrm{Nnm} / \mathrm{rad}^{2}$ which Xiao et al. [17] applied them. At last, in the model of type III, it is noted that the Morse potential can be represented by an interatomic harmonic potential similar to Eqs. (15)-(16) where the parameters $k_{r}$ and $k_{\theta}$ are obtained as $k_{r}=8.31 \times 10^{-7} \mathrm{~N} / \mathrm{nm}$ and $k_{\theta}=9 \times 10^{-10} \mathrm{Nnm} / \mathrm{rad}^{2}$ [18].

Table 1

The Young and shear modulus of SLGS with corresponding force field constants

\begin{tabular}{|c|c|c|c|c|c|}
\hline \multirow{2}{*}{$\begin{array}{c}\text { Type of } \\
\text { model }\end{array}$} & \multicolumn{3}{|c|}{ Elastic properties } & \multicolumn{2}{c|}{ Force field constants } \\
\cline { 2 - 6 } & $\begin{array}{c}E_{11}, \\
\text { TPa }\end{array}$ & $\begin{array}{c}E_{22}, \\
\text { TPa }\end{array}$ & $\begin{array}{c}G_{12}, \\
\text { TPa }\end{array}$ & $\begin{array}{c}k r, \\
\text { N/m }\end{array}$ & $\begin{array}{c}k \theta, \\
\text { nNnm/rad }\end{array}$ \\
\hline Type I & 1.131 & 1.131 & 0.424 & 652 & 0.867 \\
\hline Type II & 1.477 & 1.477 & 0.554 & 742 & 1.42 \\
\hline Type III & 1.345 & 1.345 & 0.504 & 831 & 0.9 \\
\hline
\end{tabular}

Table 2 shows the values of the stiffness coefficients of the matrix $[C]$ with respect to the force field constants. It is shown that the force field constants $k_{r}$ and $k_{\theta}$ perform a significant role in the mechanical properties of SLGS. It is seen that the stiffness coefficients depend on force field constants. The results presented in Table 2 are compared with those obtained by Tsai and Tu [5] from MD simulation and are in close agreements with them. It is seen that $v_{12}=v_{21}=0.333$. Also from Tables 1 and 2, it can be remarked that the effective properties of the structure in both $\alpha_{1}$ and $\alpha_{2}$ directions are the same (i.e., $E_{11}=E_{22}$ and $G_{12}=G_{21}$ ) and, therefore, a SLGS can be treated as a transversely isotropic material [19]. 
The stiffness matrix of SLGS calculated based on force field constants

\begin{tabular}{|c|c|c|c|c|c|}
\hline Researcher & $C_{11}=C_{22}, \mathrm{TPa}$ & $C_{12}=C_{21}, \mathrm{TPa}$ & $C_{66}, \mathrm{TPa}$ & $E_{11}=E_{22}, \mathrm{TPa}$ & $G_{12}=G_{21}, \mathrm{TPa}$ \\
\hline Present, $k_{r}$ (type I) & 0.830 & 0.277 & 0.277 & 0.738 & 0.277 \\
\hline Present, $k_{r} \& k_{\theta}$ (type I) & 1.272 & 0.424 & 0.424 & 1.131 & 0.424 \\
\hline Tsai and Tu [5] & 0.978 & 0.254 & - & 0.912 & 0.358 \\
\hline
\end{tabular}

\subsection{Bending and twisting rigidities of SLGS}

From the presented homogenization model, bending and twisting rigidities can be obtained for singlelayered graphene sheet. Upon substitution of the effective elastic coefficients from Eqs. (18)-(19) in Eq. (16) we have:

$$
\left\{\begin{array}{l}
M_{11} \\
M_{22} \\
M_{12}
\end{array}\right\}=\left[\begin{array}{ccc}
D_{11} & D_{12} & 0 \\
D_{21} & D_{22} & 0 \\
0 & 0 & D_{66}
\end{array}\right]\left\{\begin{array}{l}
\kappa_{11} \\
\kappa_{22} \\
\kappa_{12}
\end{array}\right\}
$$

From the obtained results the bending rigidity $M_{11} / \kappa_{11}$ and twisting rigidity $M_{12} / \kappa_{12}$ can be defined in terms of the bending stiffness matrix $[D]$. These rigidities are presented for single-layered graphene sheet as shown in Table 3. In this table, the present results are compared with those reported in the literature.

Table 3

Comparison of the present bending and twisting rigidities with the ones reported in the literature

\begin{tabular}{|c|c|c|c|}
\hline Researcher & Method & $\begin{array}{c}\text { Bending } D_{11}= \\
D_{22,}, \mathrm{nNnm}\end{array}$ & $\begin{array}{c}\text { Twisting } \\
D_{66}, \mathrm{nNnm}\end{array}$ \\
\hline Present & AHM & 0.402 & 0.167 \\
\hline $\begin{array}{c}\text { Kudin et al. } \\
\text { [20] }\end{array}$ & Ab initio & 0.238 & - \\
\hline Lu et al. [21] & MM & 0.225 & - \\
\hline $\begin{array}{c}\text { Kordkheili et } \\
\text { al. [22] }\end{array}$ & FEM & 0.350 & - \\
\hline
\end{tabular}

\subsection{Effect of thickness on elastic properties of SLGS}

The variations of $E_{11}$ and $G_{12}$ as functions of the thickness of SLGS are presented for $0.066 \mathrm{~nm} \leq t \leq 0.7 \mathrm{~nm}$ in Figs. 6 and 7. The values of $t$ within these limits can be found throughout the literature [15,17]. The values of $E_{11}$ and $G_{12}$, which are obtained for a single-layer graphene sheet are sensitive to varying wall thickness. It is shown in Figs. 6 and 7 that increase in wall thickness of the sheet results in a decrease of the elastic modulus. We know that in general, the thickness of a graphene sheet is considered as the distance between two adjacent graphene sheets in multi- layered graphene sheets. However, a review of the literature shows that different researchers have reported different values for Young's modulus of nanotubes or the graphene sheet and their thicknesses. Therefore, the obtained Young's modulus in the literature is not comparable. Further calculations are done from different sources; all of the numerical value of $E t$ is close. We intend to look at the reason for this discrepancy here.

In some cases, it does not need to know $E$. However, if it is necessary to calculate the exact amount of $E$ the estimated $t$ is desired. Thus, the value of $E t$ can be used as a new parameter based on which the mechanical properties of different nanotubes or the graphene sheets can be compared. Now, if $E t$ is divided by a thickness equal to the thickness of a graphene monolayer which is assumed (i.e. $0.34 \mathrm{~nm}$ ), the final values are about the same for all studied cases which are around $1 \mathrm{TPa}$. This procedure is shown in Table 4. This table summarizes and normalizes the results obtained and compares them with available results in the literature. There is not a sensible difference between the present results and those available in the literature. Such trends are in good agreement with predictions made by Scarpa et al. [7] and Georgantzinos et al. [8]. Thus, the obtained results from a viable analytical asymptotic homogenization model are reasonable, because of that, it could be applied to the other nanostructures.

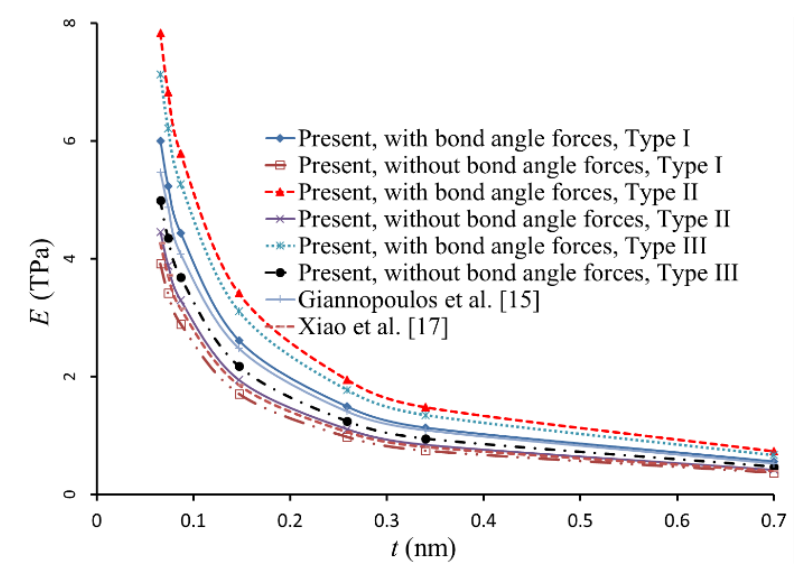

Fig. 6 Elastic modulus of SLGS to the wall thickness

Table 4

Comparison values for the reported elastic properties of the graphene sheet and their thicknesses in the literature

\begin{tabular}{|c|c|c|c|c|c|}
\hline Researcher & $t, \mathrm{~nm}$ & $v$ & $G, \mathrm{TPa}$ & $E, \mathrm{TPa}$ & $E t / 0.34, \mathrm{TPa}$ \\
\hline Present & 0.34 & 0.33 & 0.42 & 1.13 & 1.13 \\
\hline Lee et al. [2] & 0.34 & - & - & 1.00 & 1.00 \\
\hline Frank et al. [3] & 0.68 & - & - & 0.5 & 1.00 \\
\hline Tsai and $\mathrm{Tu}$ [5] & 0.34 & 0.26 & 0.36 & 0.91 & 0.91 \\
\hline Sakhaee-Pour [6] & 0.34 & 1.29 & 0.23 & 1.03 & 1.03 \\
\hline Scarpa et al. [7] - Amber & 0.34 & 0.57 & 0.21 & 1.31 & 1.31 \\
\hline Scarpa et al. [7] - Morse & 0.34 & 0.57 & 0.21 & 1.67 & 1.67 \\
\hline Georgantzinos et al. [8] & 0.34 & 0.60 & 0.28 & 1.37 & 1.37 \\
\hline Mir et al. [9] & 0.34 & - & - & 1.30 (Arm)-1.42 (Zig) & 1.30 (Arm)-1.42 (Zig) \\
\hline Kalamkarov et al. [14] & 0.14 & 0.33 & 0.32 & 1.71 & 0.74 \\
\hline
\end{tabular}




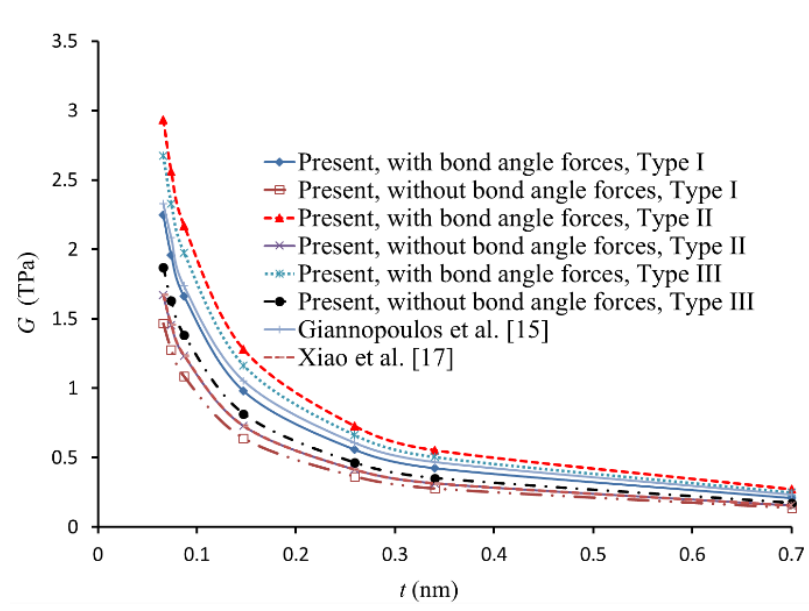

Fig. 7 The shear modulus of SLGS to the wall thickness

\section{Conclusions}

The main goal of present study is to analytically obtain the tension, bending and twisting rigidities of graphene sheet from asymptotic homogenization model. Also, Young's modulus, shear modulus and Poisson's ratio are calculated as well as rigidities of a homogenized model of SLGS. The homogenized model of SLGS consists of a honeycomb network sheet with the hexagonal unit cell, so from the analytical solution of the proper local problem in the unit cell, the effective properties of SLGS are determined. The derived formulae make easy understanding of the dependencies of SLGS mechanical properties to thickness and force field constants. It is found that the choice of covalent bond stiffnesses which are applied for stretching and bending of C-C bonds, significantly affect elastic properties of the SLGS. The predictions obtained by this model for SLGS are compared with previous theoretical and experimental results available in the literature. It is shown that the present asymptotic homogenization model not only can predict elastic properties and rigidities for SLGS but also this model can remove the difficulty of applying both stretching and bending of $\mathrm{C}-\mathrm{C}$ bonds in FE models and MD simulations. Finally, the proposed analytical method is straightforward for modeling other nanostructures with periodic structures.

\section{References}

1 Iijima, S. 1991. Helical microtubules of graphitic carbon, Nature 354: 56-58. https://doi.org/10.1038/354056a0.

2 Lee, C.; Wei, X.; Kysar, J.W.; Hone, J. 2008. Measurement of the Elastic Properties and Intrinsic Strength of Monolayer Graphene, Science 321: 385-388. https://doi.org/10.1126/science.1157996.

3 Frank, I.W.; Tanenbaum, D.M.; van der Zande, A.M.; McEuen, P.L. 2007. Mechanical properties of suspended graphene sheets, Journal of Vacuum Science \&amp; Technology B 25: 2558-2561. https://doi.org/10.1116/1.2789446.

4 Lu, Q.; Arroyo, M.; Huang, R. 2009. Elastic bending modulus of monolayer graphene, Journal of Physics Dapplied Physics 42: 102002. https://doi.org/10.1088/0022-3727/42/10/102002.

5 Tsai, J.-L.; Tu, J.-F. 2010. Characterizing mechanical properties of graphite using molecular dynamics simulation, Materials \& Design 31: 194-199. https://doi.org/10.1016/j.matdes.2009.06.032.

6 Sakhaee-Pour, A. 2009. Elastic properties of singlelayered graphene sheet, Solid State Communications 149: 91-95.

https://doi.org/10.1016/j.ssc.2008.09.050.

7 Scarpa, F.; Adhikari, S.; Phani, A.S. 2009. Effective elastic mechanical properties of single layer graphene sheets, Nanotechnology 20: 065709. https://doi.org/10.1088/0957-4484/20/6/065709.

8 Georgantzinos, S.K.; Giannopoulos, G.I.; Anifantis, N.K. 2010. Numerical investigation of elastic mechanical properties of graphene structures, Materials \& Design 31: 4646-4654. https://doi.org/10.1016/j.matdes.2010.05.036.

9 Mir, M.; Tahani, M.; Hassani, B. 2017. Analytical prediction of Young's modulus of carbon nanotubes using a variational method, Applied Mathematical Modelling 45: 1031-1043. https://doi.org/10.1016/j.apm.2017.01.038.

10 Challagulla, K.; Georgiades, A.; Kalamkarov, A. 2007. Asymptotic homogenization model for threedimensional network reinforced composite structures, Journal of Mechanics of Materials and Structures 2: 613632. https://doi.org/10.2140/jomms.2007.2.613.

11 Kalamkarov, A.L.; Andrianov, I.V.; Danishevsâ, V.V. 2009. Asymptotic homogenization of composite materials and structures, Applied Mechanics Reviews 62: 030802. https://doi.org/10.1115/1.3090830.

12 Hassani, B.; Hinton, E. 1998. A review of homogenization and topology optimization Ihomogenization theory for media with periodic structure, Computers \& Structures 69: 707-717. https://doi.org/doi.org/10.1016/S0045-7949(98)00131X.

13 Andrianov, I.V.; Danishevs'kyy, V.V.; Weichert, D. 2011. Homogenization of viscoelastic-matrix fibrous composites with square-lattice reinforcement, Archive of Applied Mechanics 81: 1903-1913. https://doi.org/10.1007/s00419-011-0526-z.

14 Kalamkarov, A.L.; Georgiades, A.V.; Rokkam, S.K.; Veedu, V.P., et al. 2006. Analytical and numerical techniques to predict carbon nanotubes properties, International Journal of Solids and Structures 43: 68326854. https://doi.org/10.1016/j.ijsolstr.2006.02.009.

15 Giannopoulos, G.; Kakavas, P.; Anifantis, N. 2008. Evaluation of the effective mechanical properties of single walled carbon nanotubes using a spring based finite element approach, Computational Materials Science 41: 561-569. https://doi.org/10.1016/j.commatsci.2007.05.016.

16 Tersoff, J. 1988. Empirical interatomic potential for carbon, with applications to amorphous carbon, Physical Review Letters 61: 2879-2882. https://doi.org/10.1103/PhysRevLett.61.2879.

17 Xiao, J.; Lopatnikov, S.; Gama, B.; Gillespie Jr, J. 2006. Nanomechanics on the deformation of single-and multi-walled carbon nanotubes under radial pressure, Materials Science and Engineering: A 416: 192-204. https://doi.org/10.1016/j.msea.2005.09.105. 
18 Belytschko, T.; Xiao, S.; Schatz, G.; Ruoff, R. 2002. Atomistic simulations of nanotube fracture, Physical Review B 65: 235430.

https://doi.org/10.1103/PhysRevB.65.235430.

19 Reddy, J.N. 2004. Mechanics of laminated composite plates and shells: theory and analysis: CRC press.

20 Kudin, K.N.; Scuseria, G.E.; Yakobson, B.I. 2001. C $2 \mathrm{~F}, \mathrm{BN}$, and $\mathrm{C}$ nanoshell elasticity from ab initio computations, Physical Review B 64: 235406. https://doi.org/10.1103/PhysRevB.64.235406.

21 Lu, Q.; Huang, R. 2009. Nonlinear mechanics of single-atomic-layer graphene sheets, International Journal of Applied Mechanics 1: 443-467. https://doi.org/10.1142/S1758825109000228.

22 Hosseini Kordkheili, S.; Moshrefzadeh-Sani, H. 2013. Mechanical properties of double-layered graphene sheets, Computational Materials Science 69: 335-343. https://doi.org/10.1016/j.commatsci.2012.11.027.
S. Safarian, M. Tahani

EVALUATION OF TENSION, BENDING AND
TWISTING RIGIDITIES OF SINGLE-LAYER
GRAPHENE SHEETS BY AN ANALYTICAL
ASYMPTOTIC HOMOGENIZATION MODEL

S u m m a r y

In the present study, the method of asymptotic homogenization is used to estimate elastic properties as well as tension, bending and twisting rigidities of single-layer graphene sheets (SLGSs). To this end, asymptotic homogenization of a reinforced composite is developed for modeling of SLGS by assuming that the covalent bond between carbon atoms can be represented by reinforcements. Applicable formulas are obtained for the elastic properties and rigidities of SLGS directly from the interatomic interactions through three types of potentials. It is proved that force field constants significantly affect the elastic properties of SLGS. Herein, the elastic moduli are obtained based on different types of atomic force constants. The results of the present analytical model are in close agreements with the similar theoretical results and experimental measurements. This approach can be developed to represent mechanical properties of nanocomposites and other nanostructures.

Keywords: Asymptotic homogenization method, Mechanical properties, Tension, bending and twisting rigidities, Single-layer graphene sheet. 